\title{
Sistema de planificación de proyectos en el desarrollo de nuevos productos en empresas de alimentos dedicadas al rubro de golosinas, 2020
}

\author{
Juan André Mendoza Castillo \\ juan.mendoza22@unmsmedu.pe \\ Universidad Nacional Mayor de San Marcos \\ Lima - Perú
}

\section{RESUMEN}

En esta investigación se implementó un sistema de planificación, con la finalidad de contribuir en el desarrollo de nuevos productos en una empresa dedicada al rubro de golosinas, 2020, la misma presentó desde el año 2019, problemas deficientes en los procesos de producción, creando incompetencias operativas y administrativas; por consiguiente, se adoptaron medidas correctivas que lograron mejorar todos los objetivos comerciales y técnicos. El tipo de investigación desarrollada es cuasi experimental porque permitió demostrar la causa-efecto de la herramienta. Los resultados muestran los efectos en reducción de tiempo respecto al desarrollo de nuevos productos pertenecientes al grupo experimental (Maquila y Full Cost). También, responde a la hipótesis general, donde el sistema de planificación influye de manera significativa en el desarrollo de nuevos productos. Además, muestra un impacto positivo al día de hoy de S/. 17,757.68, con una tasa interna de retorno de $33.37 \%$ y un beneficio costo de 2.3 soles.

Palabras clave: sistema de implementación; desarrollo de nuevos productos; golosinas; maquila; viabilidad. 


\title{
Project planning system in the development of new products in food companies dedicated to the candy industry, 2020
}

\begin{abstract}
In this research, a planning system was implemented, in order to contribute to the development of new products in a company dedicated to the candy industry, 2020, it presented since 2019, deficient problems in the production processes, creating incompetence operational and administrative; consequently, corrective actions were taken that were successful in improving all business and technical objectives. The type of research developed is quasi-experimental because it allowed to demonstrate the causeeffect of the tool. The results show the effects in time reduction with respect to the development of new products belonging to the experimental group (Maquila and Full Cost). Also, it responds to the general hypothesis, where the planning system has a significant influence on the development of new products. In addition, it shows a positive impact to date of S /. 17,757.68, with an internal rate of return of $33.37 \%$ and a cost benefit of 2.3 soles.
\end{abstract}

Keywords: implementation system; development of new products; sweets; maquila; viability. 


\section{INTRODUCCIÓN}

Las empresas de alimentos dedicadas al rubro golosinario tienen como objetivo alcanzar el nivel estándar de las grandes compañías de alimentos en el mundo. En este contexto, Implementar un plan de ejecución que influya en el desarrollo de nuevos productos en una empresa de alimentos dedicada al rubro de golosinas es considerada una necesidad, puesto que las empresas buscan ser altamente competitivas en el mercado, siendo necesario, desarrollar buenas prácticas de manufactura que contribuyan al buen funcionamiento de las plantas de proceso. Las empresas del rubro alimenticio actualmente no solo fabrican productos para colocarlos en el mercado y esperar que cubran las expectativas del consumidor. Hoy en día la mayoría de las empresas de alimentos dedicadas al rubro de golosinas tienen como objetivo alcanzar el nivel estándar de las grandes compañías de alimentos en el mundo.

Al iniciar la planificación de proyectos la cual influye en el tiempo de desarrollo de nuevos productos, se tiene por objetivo obtener la información de las actividades a desarrollar para lograr las metas trazadas. Los proyectos relacionados a la industria de alimentos se orientan a mejorar de manera constante el proceso de transformación de alimentos. Otro de los principales objetivos de las empresas de productos golosinarios es satisfacer las necesidades nutricionales de cierto grupo etario de la población, como es el caso de la empresa Nestlé, Alicorp y otras grandes empresas que se extienden a lo largo del país llegando a millones de consumidores en todo el territorio nacional, convirtiéndolas en empresas líderes a nivel nacional con acceso al mercado mundial.

El rubro de golosinas tiene gran demanda de consumo en el Perú; muchas empresas locales dedicadas a este rubro compiten entre ellas y contra grandes empresas transnacionales para lograr posicionarse de la preferencia de los consumidores. En el caso de Nestlé, por citar un ejemplo; es una empresa que se ha posicionado en la preferencia de los consumidores a través de sus productos golosinaros entre los que destacan los chocolates: Sublime, Princesa, Triángulo, Lentejas, Beso de Moza las cuales son marcas clásicas que la han convertido en el líder absoluto de su rubro, logrando que sus productos sean de gran calidad y sobre todo accesibles para todos los consumidores.

Las empresas de este rubro que ya cuentan con prestigio a nivel mundial han logrado alcanzar un alto nivel de competitividad en el mercado, enfocándose en las buenas prácticas de manufactura, desarrollando proyectos de mejora continua que incrementen 
la productividad y rendimiento de sus operaciones, estableciendo para ello un modelo de gestión de proyectos.

Gómez et al. (2009) en su libro Manual de Gestión de Proyectos sostienen que la gestión de proyectos es el enfoque primordial del trabajo, el cual no es posible adaptarlo sin tomar en cuenta cómo las instituciones organizan sus recursos y desarrollan un conjunto de actividades. Siendo propuestas para orientar el desempeño de la empresa. Para Cruz et al. (2018) en su tesis refiere que se debe manejar los recursos de manera eficiente (materia prima, recursos económicos, recursos humanos) que el desarrollo de buenas prácticas de manufactura al buscar mejora continua en las empresas, lo cual debe ser un objetivo constante en el que los directivos de las empresas y sus colaboradores deben comprometerse e identificarse para el logro del éxito de la empresa. Seguidamente, Lavaggi, Mori y Rozas (2016) sostienen que desarrollar un plan de negocios es enfocarse en la viabilidad de la exportación del producto. Basaron sus estudios en la exportación de chocolate orgánico a mercados competitivos como es el caso de Estados Unidos. Es así como Martel, Peña, Ramos y Silva Santisteban (2015), sostienen que las empresas del rubro de alimentos entre los que se encuentran los productos golosinarios, como el caso de Alicorp S.A.A son altamente competitivas porque se basan en el precio del producto, la constante innovación, la calidad de sus productos y principalmente la capacidad de identificar y satisfacer las necesidades de los consumidores. Desde esta línea, la Justificación de la Investigación, busca mediante el conocimiento conceptual del sistema de planificación, hallar razones vinculadas a la influencia que pueda tener sobre el desarrollo de nuevos productos en la empresa de alimentos golosinarios; ya que en el Perú existen empresas del rubro alimenticio que elaboran diversos productos golosinarios que numerosas veces surgen desde el entorno familiar, los cuales vienen evidenciando deficiencias en cuanto a los proceso de producción así como en sus estrategias comerciales y de ventas, Siendo por ello necesario determinar el grado de influencia, con la finalidad de detectar las deficiencias operativas y administrativas que en dichas empresas puedan estar incurriendo; Así como establecer los procedimientos necesarios, adoptando medidas correctivas que logren mejorar su nivel productivo, sus estrategias comerciales y de ventas que le permitan posicionarse de manera exitosa en el mercado competitivo. basado en el nivel de ventas de sus productos. Es así como la aplicación del sistema de planificación para optimizar la elaboración de nuevos productos golosinarios, 
mientras que los demás colaboradores (Específicamente del área de producción) deben estar comprometidos con el crecimiento de la empresa, asimismo se utilizarán instrumentos validados para evaluar el sistema de planificación de proyectos y su influencia en el tiempo de desarrollo de nuevos productos en empresas de alimentos dedicadas al rubro golosinario, para el periodo 2021.

\section{Sistema de Planificación}

La planificación plantea varios elementos epistemológicos: el sujeto que planifica; el espacio poblacional sobre el que se toman las decisiones; los marcos de trabajo y la utilización del conocimiento científico mediante la cual se selecciona la evidencia (objetivo/ abstracto vs. subjetivo/experiencial) (Lopera, 2014). De acuerdo con la Organización Internacional de Trabajo (2016) el sistema de planificación es útil para incrementar las actividades del negocio, incrementar las ventas y reducir costos. Es así como el sistema de planificación brinda a la empresa información confiable y de calidad que contribuye a una adecuada toma de decisiones en la empresa y a ejercer medidas de supervisión del negocio. Por su parte Vilcapoma (2016) sostiene que un adecuado sistema de planificación también evalúa el cumplimiento de objetivos entre los cuales tenemos mejora de productividad, mejora de márgenes de utilidad, cumplimiento de satisfacción a clientes y/o consumidores, eficiencia y eficacia en el proceso productivo, entre otros. Seguidamente se manifiesta las subcategorías que ha mostrado el efecto positivo, y cumpliendo nuestro propósito, que es el objetivo general y específicos de la investigación. A) Estrategia. es un Sistema de Planificación y Control de la Producción para mejorar el nivel de servicio sostiene que la identificación de problemas en el proceso productivo presenta causas desde el punto de vista metodológico y logístico. B) Exploración. Lleva a cabo la validación del producto y confirmar la viabilidad, capacidad para industrializar e iniciar la demanda. C) Ejecución. Son indicadores que preparan principalmente un caso de negocio y detalle el plan del proyecto. D) Entrega. - Es la mayor planeación para nuevos servicios en el lanzamiento de nuevos productos físicos. (Vera, 2018)

\section{Desarrollo de Nuevos Productos}

Es buscar como de forma organizada se puede llegar a un resultado que se dio en primera instancia por una incógnita, una necesidad inicial, cuando se conoce el resultado se da fin a ese proyecto, y tal vez, se puede empezar otro (Estrada, 2015). Cada industria posee su forma particular de gestionar sus proyectos, claro que existen lineamientos 
internacionales estandarizados como los que muestra la guía del PMBOK, la cual tienen libre albedrio de seguir o no. Aplicado a proyectos cortos las guía PMBOK recomienda usar solo las áreas del conocimiento que comprendan o se toquen en el proyecto, así como los formatos y entregables que hagan más sencillo y concreto el avance y desarrollo del proyecto. Para Távara, (2018) quien sostiene que, con el desarrollo del Acta de Constitución del Proyecto, es necesario definir los parámetros de tiempos y costos dentro de un Proyecto. Asimismo, Valderrama, (2018); estandariza su proceso productivo logrando incluso reducir costos. A su vez sostiene, el aplicar la filosofía Lean Manufacturing siendo favorable en las empresas al estar encaminada directamente a mejorar la competitividad creando el máximo valor agregado al consumidor. Por su parte Contreras y Palacios, (2017), en corriente se concentró en el área de los inventarios en un $50 \%$ del activo corriente. También San Román \& Valdizán, (2017) empleó el método aplicado siendo positivo el desarrollo de los modelos financieros y económicos del proyecto. Seguidamente se muestran las subcategorías analizadas en la investigación, siendo una metodología aplicada hasta el año 2019, conformada por el grupo control siendo: A) Sistema de fijación de tiempos de entrega. -Se define como el lead time (Tiempo de entrega) es el tiempo que trascurre entre el pedido del cliente y la llegada del producto al cliente final (Pérez, 2016). B) Tiempo y cantidad en el desarrollo de procesos de un proyecto. -Es el proyecto que posea un plazo viable para ejecutar la entrega del resultado esperado del Proyecto (Hito Master DAP). C) La optimización y estandarización de procesos. -Es aquella que mejora y/o simplifica los procesos de las instituciones de la APF se facilita la obtención, entre otros, de los siguientes resultados: (Secretaría de la Función Pública). D) El tiempo de atención y cantidad de proyectos terminados. -Cumple una función de necesidades de gestión y control de la organización $\mathrm{u}$ organizaciones que participan en el proyecto, la naturaleza propia del proyecto y su área de aplicación (Umaña, 2016).

\section{METODOLOGÍA}

En el presente estudio, se midieron las variables desde el enfoque cuantitativo, en donde la investigación desarrolla un estudio de tipo cuasi experimental por que se manipulará la variable independiente. Al respecto "Una investigación es experimental cuando en una situación de control se va a manipular en forma intencional una o varias variables 
independientes para estudiar sus efectos en otras dependientes". (Hernández et al., 2014, p. 32)

En la investigación se estudió el efecto de la aplicación del sistema de planeación, con el fin de mejorar el desarrollo de nuevos productos, en el presente y por consiguiente a futuro.

\section{Hipótesis}

Muestra las hipótesis y las variables dependientes e independientes desarrolladas en la presente investigación.

\section{Tabla 1 Hipótesis y variables}

\begin{tabular}{ll}
\hline \multicolumn{1}{c}{ Hipótesis general } & \multicolumn{1}{c}{ Variables } \\
\hline $\begin{array}{l}\text { La Implementación de un sistema de planificación } \\
\text { influye de manera directa en el desarrollo de nuevos }\end{array}$ & VI: Sistema de Planificación \\
productos en una empresa de golosinas & Vesarrollo de Nuevos Productos \\
\hline \multicolumn{1}{c}{ Hipótesis específicas } & \multicolumn{1}{c}{ Subvariables } \\
\hline $\begin{array}{l}\text { El sistema de Estrategia influye de manera directa en } \\
\text { fijación de tiempos de entrega en una empresa de }\end{array}$ & VD: Tiempos de entrega \\
golosinas & \\
$\begin{array}{l}\text { El Sistema de Exploración influye de manera directa } \\
\text { en tiempo y cantidad de desarrollo de procesos de un }\end{array}$ & VD: Tiempo y cantidad de desarrollo de \\
proyecto en una empresa de golosinas & procesos de un proyecto \\
El Sistema de Ejecución influye de manera directa & VI: Sistema de Ejecución \\
en optimización y estandarización de procesos, en & VD: Optimización y estandarización de \\
una empresa de golosinas & procesos \\
$\begin{array}{l}\text { El Sistema de Entrega influye de manera directa en } \\
\text { tiempo de atención y cantidad de proyectos }\end{array}$ & VI: Sistema de Entrega \\
terminados en una empresa de golosinas & VD: Tiempo de atención y cantidad de
\end{tabular}

Fuente: Elaborado por el investigador.

\section{Población de estudio}

La población, tiene por finalidad, mostrar el conjunto de datos que conforman un estudio, estratificado o aleatoria. (Hernández et al. 2014, p. 250); Desde esta línea la presente investigación son $\mathrm{p}=325$ proyectos, pertenecientes a las familias de chocolates en barra, chocolates en bloque y chocolates cobertura en una empresa de golosinas categorizados en productos innovadores, de reemplazo, extensión de líneas, imitadores, mejorados y de reposicionamiento de mercado. 


\section{Tamaño de muestra}

Desde esta línea, para hallar la muestra y esta sea probabilística se aplica la siguiente formula:

$$
n=\frac{N Z^{2} P Q}{d^{2}(N-1)+Z^{2} P Q}
$$

De acuerdo con el resultado obtenido de la formula, la muestra está contempla la cobertura de una empresa de golosinas de marcas reconocidas tales como Nestlé S.A, Móndeles S.A, Alicorp, Arcor, entre otros y en la actualidad la suma de proyectos asciende a más de 140 proyectos.

\section{Técnicas de recolección de Datos}

Los datos, para el grupo experimental siendo $n=70$ proyectos, la ficha técnica Lista de cotejo, por cada etapa. Sin embargo, para el grupo de control $n=70$, se tomó como técnica ficha de observación, lista de cotejo, según las etapas, desarrolladas por los especialistas en el año 2019.

\section{Validez}

Los instrumentos, fueron validados por tres expertos, en la materia, con el fin de reforzar la herramienta como tal en el proceso de mejora en cuanto al cumplimiento de las etapas, para un eficaz y lo más importante eficiente desarrollo de productos nuevos en una empresa de golosinas.

\section{Confiabilidad}

Los instrumentos validados por los expertos, se le aplicó el estadístico K Richardson, para determinar la fiabilidad de los tableros como fichas de cotejo y las fichas de respuesta a la observación realizada durante todo el proceso de desarrollo de nuevos productos, con la implementación del sistema de planificación.

\section{Análisis de la información}

Análisis de lo situación actual, con respecto al desarrollo de nuevos productos durante el periodo 2019, que fueron desarrollados 70 proyectos, de los cuales se evidencio que más de la mitad de los proyectos se entregaron a destiempo, por lo que se hizo un análisis minucioso, detectándose la oportunidad de implementar una herramienta de planificación de gestión de proyectos que permitiera lograr entregar los nuevos productos en tiempo, logrando así satisfacer la necesidad de entrega al cliente en la fecha pactada cumpliendo 
con los requisitos de calidad y seguridad alimentaria. Dicho de otra manera, el desarrollo de nuevos productos se evidencia que de los 70 .

Proyectos aceptados durante el año 2019 (Muestra), llámese “Grupo control” solo el $27.14 \%$ de los proyectos se entregaron a tiempo, el $68.57 \%$ se entregaron a destiempo y el 4.29\% no se llegó a entregar, generando una insatisfacción en el cliente al no completar con el servicio por inconvenientes en la planificación del proyecto. En efecto el $47.14 \%$ de los proyectos se detuvieron en la Etapa 2 "Tiempo de desarrollo de proyectos", el $21.43 \%$ se detuvo en la Etapa 3 "Cantidad de procesos de un proyecto", el 20\% se detuvo en la Etapa 4 "Optimización y estandarización de procesos", el 5.71\% se detuvo en la Etapa 5 “Tiempo de atención al cliente", el 4.29\% se detiene en la Etapa 1 "Sistema de fijación de tiempos de entrega", llegando a la Etapa final la Etapa 6 "Proyectos terminados" tan solo un 1.43\%. Es decir que en la Etapa 2 donde casi el 50\% de los proyectos se detienen al no tener un método de planificación robusto.

Figura 1. Tiempo de desarrollo del proyecto (proceso antes)

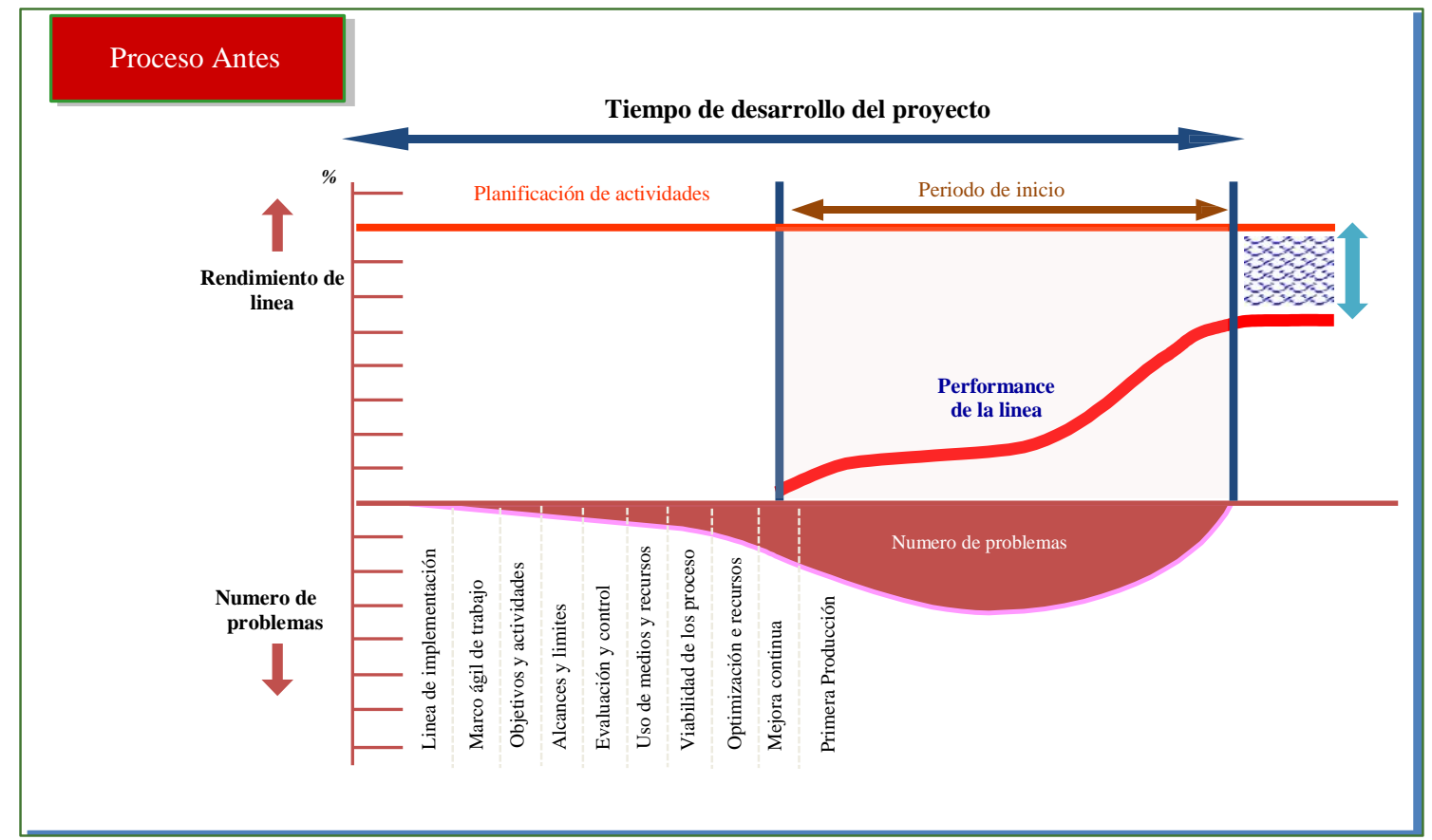

Fuente: Elaborado por el investigador en base a los datos del año 2019.

La Figura 1, muestra el rendimiento desde una línea vertical, desde punto ascendente, obteniendo un $70 \%$ de performance y un $30 \%$ oportunidad. Dicho de otra manera, esta figura evidencia que este sistema es más reactivo, puesto que se dedica a corregir errores. Seguidamente, el sistema de fijación de tiempo de entrega, según las necesidades de desarrollar 70 proyectos, alcanzo a entregar a destiempo 48 proyectos, y 3 sin entregar; 
estos tres últimos, generan insatisfacción en los clientes, siendo la causa principal la deficiencia en la fijación de tiempos. Para el tiempo de desarrollo y la cantidad de procesos se encontró que, de los 70 proyectos desarrollados, 48 fueron entregado a destiempo y 3 no se entregaron, generando también insatisfacción en los clientes; dado que causa una deficiencia en el tiempo de desarrollo y en la cantidad de procesos de un proyecto. Seguidamente la optimización y estandarización de los procesos, se suma las necesidades encontradas dentro de los 70 proyectos desarrollados, 48 fueron entregados a destiempo y 3 no se entregaron. Asimismo, generando insatisfacción en los clientes, siendo la causa principal, la deficiencia en la optimización y en la estandarización de procesos. Para finalizar con estas evidencias, de los 70 proyectos con respecto al tiempo de atención y cantidad de proyectos terminados, solo 5 proyectos cumplieron esta dimensión.

Sin embargo, para el resto genero una insatisfacción siendo causa principal la deficiencia en el tiempo de atención y cantidad de proyectos terminados.

Es así como se detecta los hallazgos durante el periodo 2019 en los 70\% proyectos de desarrollo de nuevos productos. Y donde nace la necesidad de transformarlo en el periodo 2020, mediante la aplicación de un KPI Transformador, el cual permitirá emplear las herramientas que ayude a la mejorar los hallazgos antes mencionados, mediante la aplicación de un "Sistema de Planificación", con 70 nuevos proyectos, con la finalidad de cumplir con el objetivo, siendo necesario trabajar en base a las sus cuatro etapas “Estrategia, donde está el punto de quiebre, para así planear, el proceso siguiente que es Exploración, seguidamente logrando la segunda etapa, en donde se ubican los puntos críticos de los proyectos desarrollados durante el periodo antes expuesto, se logra con el cumplimiento de la misma y pasando a la etapa Ejecución, dicho de otra manera, esta etapa en el tiempo planeado, logra concluir $99 \%$ de los proyectos, a fin de cumplir la última etapa que es entrega en el tiempo y estandarizado por el sistema de planificación y por consiguiente se logra con los clientes la entrega de los proyectos terminados. 
Figura 2. Tiempo de desarrollo de proyectos (Procesos después)

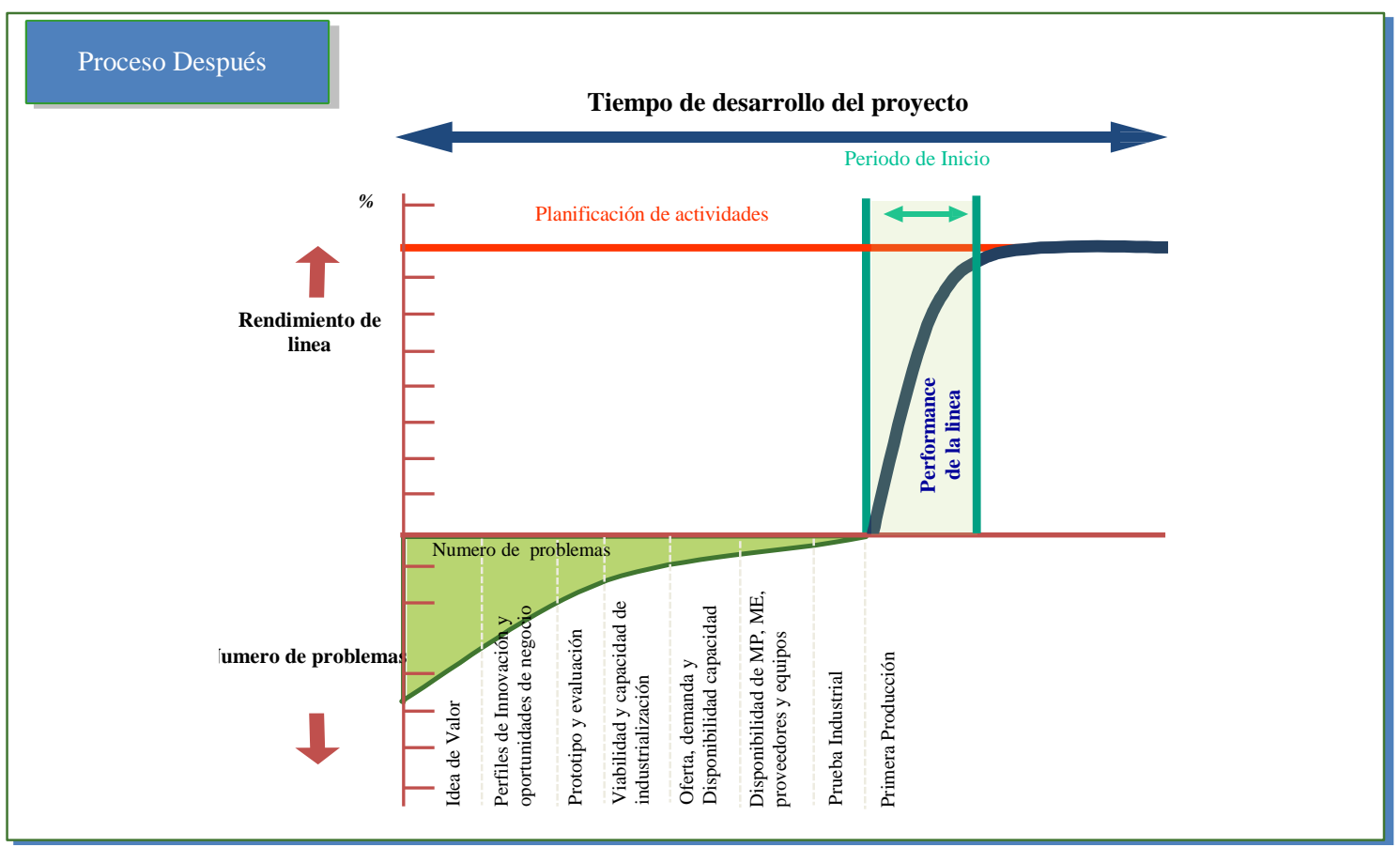

Fuente: Elaborado por el investigador en base a los datos del año 2020.

La Figura 2, muestra un performance del 100\%, optimizando el tiempo de entrega de los proyectos terminados. Después de haberse implementado el sistema de planificación en la gestión del desarrollo de nuevos productos se evidencia que, de los 70 proyectos aceptados durante el 2020, llámese "Grupo experimental" el 79\% de los proyectos se entregaron a tiempo y solo el $21 \%$ presento un retraso en la entrega, cabe resaltar que ningún desarrollo dejo de atenderse, logrando además cumplir con lo prometido al cliente y alcanzando los objetivos de la compañía.

Después de todo el análisis, de las nuevas 4 etapas consignadas, el 50\% de los proyectos llegaron a la última etapa, la Etapa 4 "Entrega", el 28.6\% se detuvo en la Etapa 3 "Ejecución", el 21.4\% se detuvo en la Etapa 3 "Exploración"; Cabe indicar que, en la etapa de cada proyecto, todos llegaron a ser atendidos, lográndose un gran índice de satisfacción de nuestros clientes. Concluyéndose que al implementar el sistema de planificación se incrementó el índice de proyectos terminados a tiempo de un 19\% a un $55 \%$, que la cantidad de proyectos demorados se redujo de un $48 \%$ a tan solo un $15 \%$ y que no se volvieron a presentar proyectos no terminados o inconclusos 
Figura 3. Reuniones Operacionales

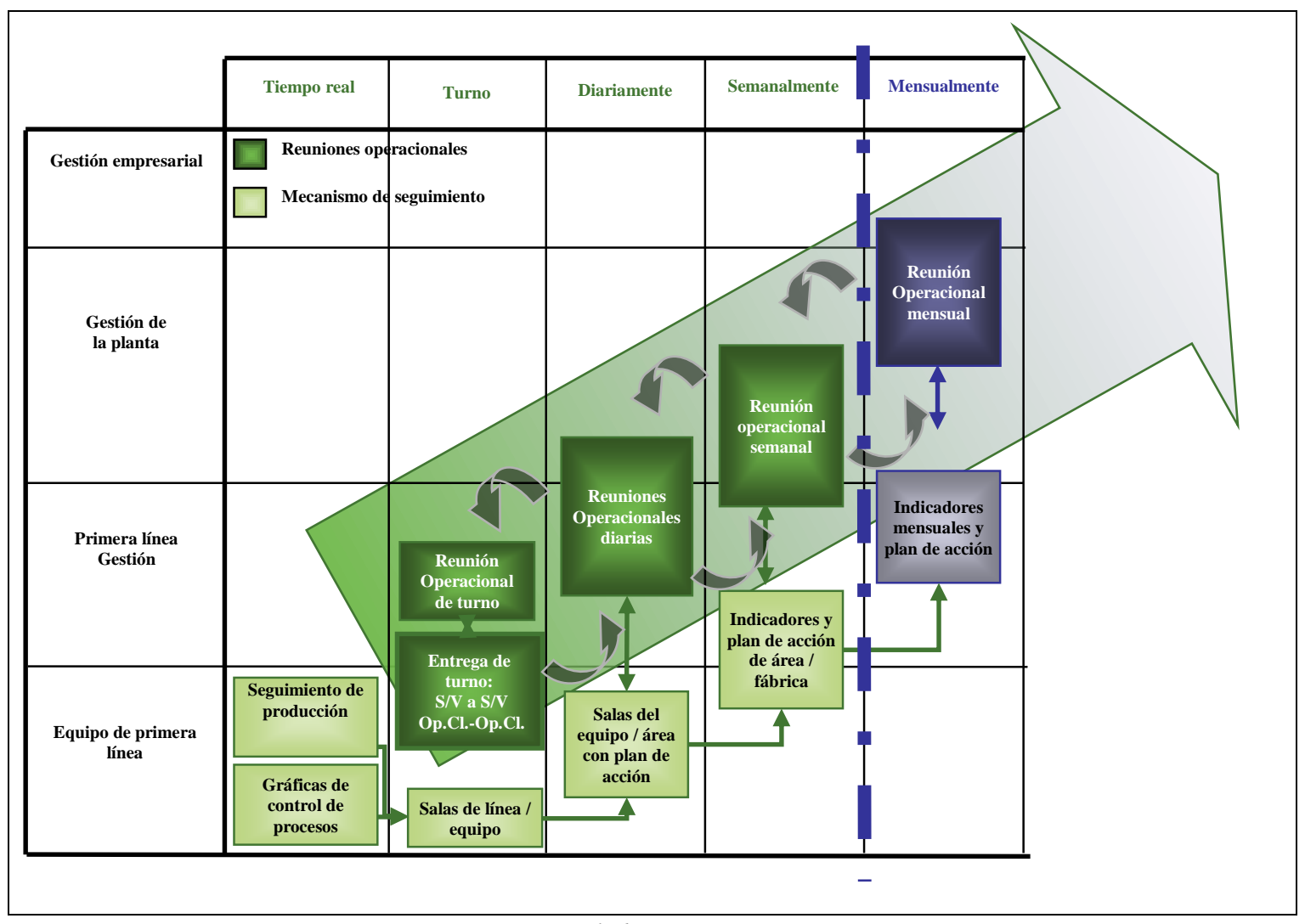

Fuente: Elaboración Propia

\section{RESULTADOS Y DISCUSIÓN}

La Figura 4. Muestra a escala la distribución del comparativo entre los Grupo Control y Experimental según la categoría donde se detienen de los proyectos, evidenciándose la eficacia de la herramienta actual para la gestión de proyectos.

\section{Figura 4. Grupo Control vs Grupo Experimental}

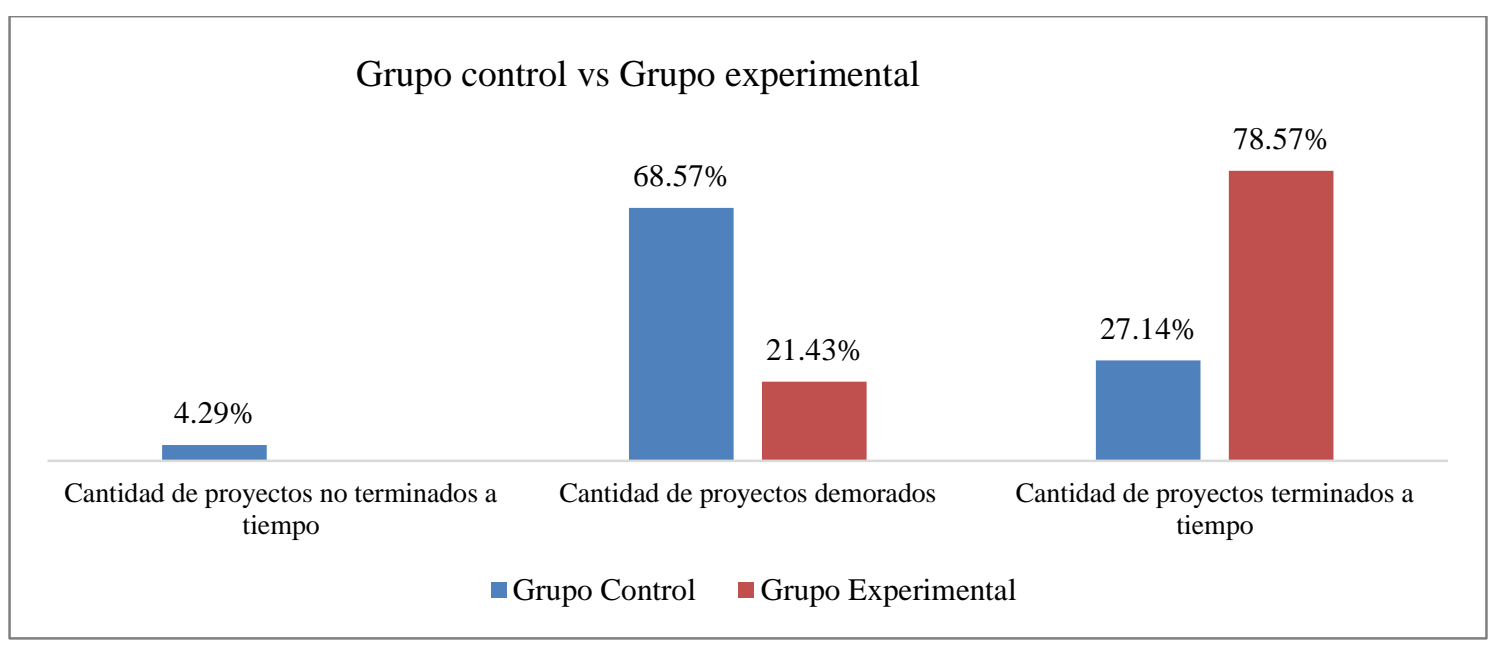

Fuente: Base de datos 2019 - 2020 Proyectos de producto de Chocolatería 
Figura 5. Startup Operacional 9 pasos y 28 actividades

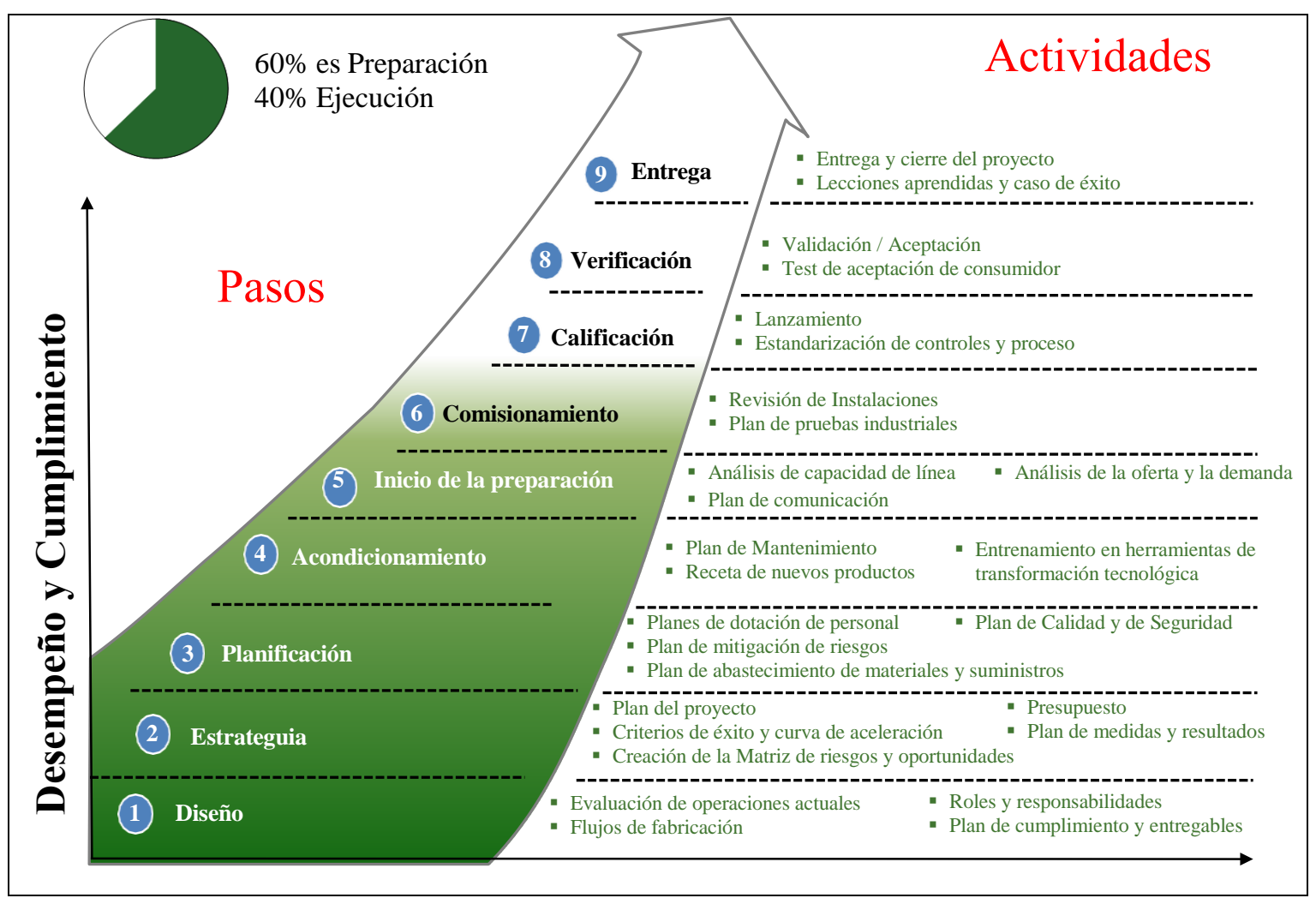

Fuente: Elaboración propia

La Figura 5. Muestra los pasos y actividades claves para la gestión de proyectos

\section{Pruebas de hipótesis}

El procedimiento que se llevó a cabo para la comprobación de las hipótesis fue en base a la prueba estadística denominada $\mathrm{t}$ de Student, determinadas previo a la prueba de normalidad, encontrando que los datos provienen de una distribución normal, por tanto, el análisis cumple con el supuesto en donde el Sistema de Planificación como herramienta se soporta de manera normal el procedimiento para cumplir con el objetivo previsto.

\section{Hipótesis General}

Ha. La Implementación de un sistema de planificación influye de manera directa en el desarrollo de nuevos productos en una empresa de golosinas.

Ho. La Implementación de un sistema de planificación NO influye de manera directa en el desarrollo de nuevos productos en una empresa de golosinas. 
Tabla 2. Prueba $t$-Student

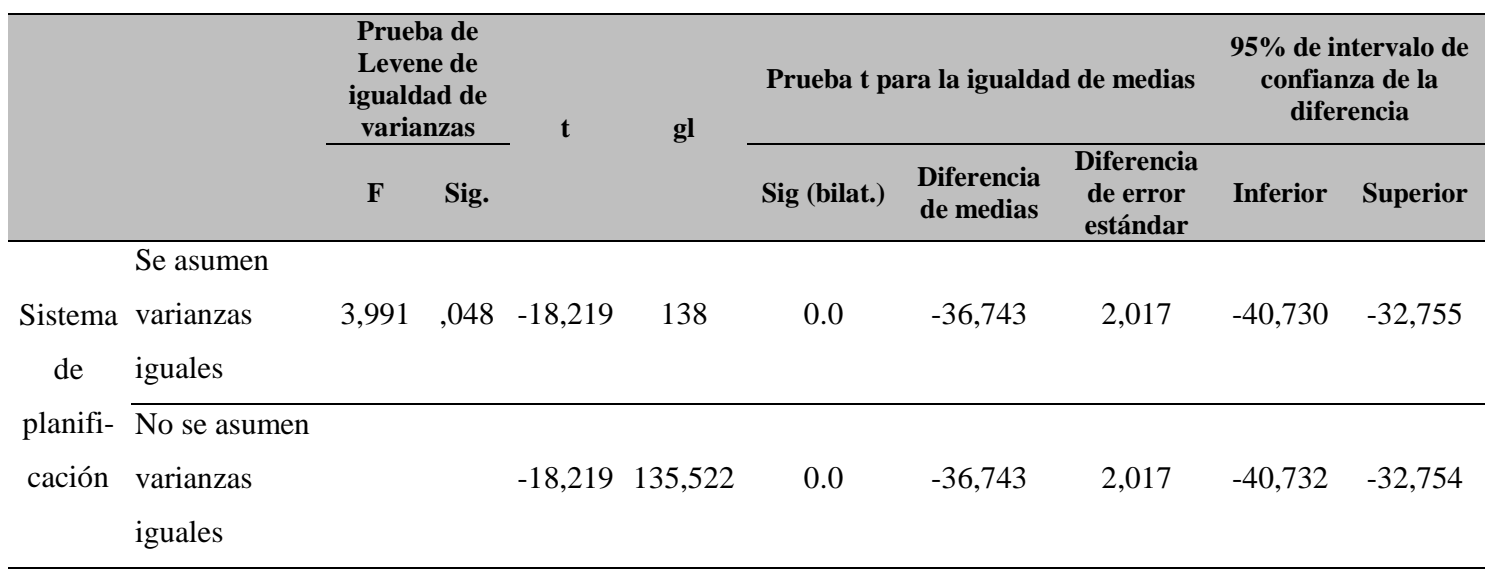

Fuente: Elaboración propia

\section{Decisión estadística}

Se evidencia en los resultados obtenidos en la prueba T-students, en cuanto al Sistema de Planificación en el grupo de control como experimental; se refleja que existe un nivel de significancia positivo siendo $0.048<0.05$; Asimismo $\mathrm{t}_{\text {obtenido }}-18,219$; responde a la hipótesis alterna que La Implementación de un sistema de planificación influye de manera directa en el desarrollo de nuevos productos en una empresa de golosinas, Periodo, 2020.

\section{Hipótesis Específica 1}

Ha. La implementación del sistema estrategia influye de manera directa en el desarrollo de nuevos productos según el Sistema de fijación de tiempos de entrega en una empresa de golosinas.

Ho. La implementación del sistema estrategia NO influye de manera directa en el desarrollo de nuevos productos según el Sistema de fijación de tiempos de entrega en una empresa de golosinas.

Tabla 3. Prueba de muestra independiente de factor Bayes $(\text { Método }=\text { Rouder })^{a}$

\begin{tabular}{lc|c|c|c|c|c}
\hline & $\begin{array}{c}\text { Diferencia de } \\
\text { medias }\end{array}$ & $\begin{array}{c}\text { Diferencia de } \\
\text { error estándar } \\
\text { agrupada }\end{array}$ & Factor Bayes $^{\mathbf{b}}$ & t & df & Sig.(bilateral) \\
\hline Estrategia &, 70 &, 844 & 5,484 &, 829 & 138 &, 408 \\
\hline
\end{tabular}

a. Asume una varianza distinta entre grupos.

b. Factor Bayes: hipótesis nula versus hipótesis alternativa

Fuente: Elaboración propia 


\section{Decisión estadística}

Se evidencia en los resultados obtenidos en la prueba T-students, en cuanto al Sistema de Planificación en el grupo de control como experimental; se refleja que existe un nivel de significancia positivo siendo $0.408>0.05$; Asimismo tobtenido, 844 ; responde a la hipótesis nula que la implementación del sistema estrategia NO influye de manera directa en el desarrollo de nuevos productos según el Sistema de fijación de tiempos de entrega en una empresa de golosinas, Periodo, 2020.

\section{Hipótesis Específica 2}

Ha. La implementación del sistema de exploración influye de manera directa en el desarrollo de nuevos productos según tiempo y cantidad en el desarrollo de procesos de un proyecto en una empresa de golosinas.

Ho. La implementación del sistema de exploración NO influye de manera directa en el desarrollo de nuevos productos según tiempo y cantidad en el desarrollo de procesos de un proyecto en una empresa de golosinas.

Tabla 4. Prueba de muestra independiente de factor Bayes $(\text { Método }=\text { Rouder })^{a}$

\begin{tabular}{lcc|c|c|c|c}
\hline & $\begin{array}{c}\text { Diferencia de } \\
\text { medias }\end{array}$ & $\begin{array}{c}\text { Diferencia de error } \\
\text { estándar agrupada }\end{array}$ & Factor Bayes $^{\mathbf{b}}$ & $\mathbf{t}$ & df & Sig.(bilatera) \\
\hline Exploración & 11,87 &, 818 &, 000 & 14,508 & 138 &, 000 \\
\hline
\end{tabular}

a. Asume una varianza distinta entre grupos.

b. Factor Bayes: hipótesis nula versus hipótesis alternativa

\section{Fuente: Elaboración propia}

\section{Decisión estadística}

Se evidencia en los resultados obtenidos en la prueba T-students, en cuanto al Sistema de Planificación en el grupo de control como experimental; se refleja que existe un nivel de significancia positivo siendo $0.000<0.05$; Asimismo $t_{\text {obtenido }} 14,508$; responde a la hipótesis alterna que la implementación del sistema de exploración influye de manera directa en el desarrollo de nuevos productos según tiempo y cantidad en el desarrollo de procesos de un proyecto en una empresa de golosinas, Periodo, 2020.

\section{Hipótesis Específica 3}

Ha. La implementación del sistema de ejecución influye de manera directa en el desarrollo de nuevos productos según la optimización y estandarización de procesos, en una empresa de golosinas. 
Tabla 5. Prueba de muestra independiente de factor Bayes $(\text { Método }=\text { Rouder })^{a}$

\begin{tabular}{|c|c|c|c|c|c|c}
\hline & $\begin{array}{c}\text { Diferencia de } \\
\text { medias }\end{array}$ & $\begin{array}{c}\text { Diferencia de } \\
\text { error estándar } \\
\text { agrupada }\end{array}$ & $\begin{array}{c}\text { Factor } \\
\text { Bayes }^{\mathbf{b}}\end{array}$ & t & df & Sig.(bilateral) \\
\hline Ejecución & 15,56 &, 857 &, 000 & 18,157 & 138 &, 000 \\
\hline
\end{tabular}

a. Asume una varianza distinta entre grupos.

b. Factor Bayes: hipótesis nula versus hipótesis alternativa

Fuente: Elaboración propia

\section{Decisión estadística}

Se evidencia en los resultados obtenidos en la prueba T-students, en cuanto al Sistema de Planificación en el grupo de control como experimental; se refleja que existe un nivel de significancia positivo siendo $0.000<0.05$; Asimismo $t_{\text {obtenido }} 18,157$; responde a la hipótesis alterna que la implementación del sistema de ejecución influye de manera directa en el desarrollo de nuevos productos según la optimización y estandarización de procesos, en una empresa de golosinas, Periodo, 2020.

\section{Hipótesis Específica 4}

Ha. La implementación del sistema de Entrega influye de manera directa en el desarrollo de nuevos productos según el tiempo de atención y cantidad de proyectos terminados en una empresa de golosinas.

Ho. La implementación del sistema de Entrega NO influye de manera directa en el desarrollo de nuevos productos según el tiempo de atención y cantidad de proyectos terminados en una empresa de golosinas.

Tabla 6. Estadísticas de grupo

\begin{tabular}{lll|l|c|c}
\hline & \multicolumn{1}{c}{ Grupo } & $\mathbf{N}$ & Media & Desv. Desviación & Desv. Error promedio \\
\hline \multirow{2}{*}{ Entrega } & $=$ Control & 70 & 12,44 & 1,953 &, 233 \\
\cline { 2 - 6 } & $=$ Experimental & 70 & 20,84 & 2,811 &, 336 \\
\hline
\end{tabular}

Fuente: Elaboración propia 
Tabla 7. Prueba de muestra independiente de factor Bayes (Método $=$ Rouder) ${ }^{a}$

\begin{tabular}{lc|c|c|c|c|c}
\hline & \multicolumn{2}{c}{$\begin{array}{c}\text { Diferencia Diferencia de error } \\
\text { de medias estándar agrupada }\end{array}$} & Factor Bayes & t & df & Sig.(bilateral) \\
\hline Entrega & 8,40 &, 409 &, 000 & 20,531 & 138 &, 000 \\
\hline
\end{tabular}

a. Asume una varianza distinta entre grupos.

b. Factor Bayes: hipótesis nula versus hipótesis alternativa

Fuente: Elaboración propia

\section{Decisión estadística}

Se evidencia en los resultados obtenidos en la prueba T-students, en cuanto al Sistema de Planificación en el grupo de control como experimental; se refleja que existe un nivel de significancia positivo siendo $0.000<0.05$; Asimismo $t_{\text {obtenido }} 20,531$; responde a la hipótesis alterna que la implementación del sistema de entrega influye de manera directa en el desarrollo de nuevos productos según el tiempo de atención y cantidad de proyectos terminados en una empresa de golosinas, Periodo, 2020.

\section{DISCUSIÓN}

El presente estudio de investigación ha demostrado en diferentes escenarios, la eficacia de la propuesta aplicada en la presente investigación en cuestión a la implementación del sistema de planificación, encontrando resultados directos y positivos. Sin embargo, en la hipótesis específica uno, no se logró encontrar diferencias significativas, por la misma razón es que los ingenieros son especialistas en el tema, materia de estudio desarrollo de nuevos productos. Sin embargo, en el proceso de su desarrollo y/o ejecución durante los años anteriores, no cumplían con las expectativas de sus clientes, encontrando disconformidad respecto a los tiempos de entrega en específico. Asimismo, en la implementación de este sistema de planificación, que responde a nuestra hipótesis general planteada, responde a la hipótesis positiva donde indica que "La Implementación del sistema de planificación influye de manera directa en el desarrollo de nuevos productos en una empresa de golosinas, Periodo, 2020”, Por consiguiente, otros estudios responden como es el caso de Uribe, (2018) en su investigación, implementó la Metodología Pmbok en lo Referente en Tiempo y Costo; en donde concluye que la baja incorporación de la RSE en la GP; la poca utilización de estándares, metodologías o prácticas sistemáticas de GP; y la conveniencia para el sector de adoptar un modelo de gestión de la RSE en el ámbito de los proyectos, como el que se ha diseñado, con base en el enfoque de procesos 
y la cadena de valor. Por su parte Cabrera (2018) en su investigación analiza y concluye la necesidad de una factibilidad financiera, económica y técnica de la empresa productora de jugos, con el fin de cumplir un rol importante para la puesta en marcha de una entidad. De la misma forma Salinas (2016); en su investigación refiere que la industrialización y comercialización del chocolate cuenta con marketing excesivo, por lo que considera importante y necesario, aplicar estrategias que permitan un buen procesos de elaboración de elaborar chocolates sin adicionar elementos químicos ni sintéticos; ya que estudios científicos han demostrado que el chocolate tipo raw elaborado con cacao ecológico dentro de su aporte nutricional contiene anti oxidantes que favorecen al consumidor en cuanto a la salud cardiovascular. De forma similar Varela, (2015), en su investigación, hace hincapié la importancia del proceso de innovación, en lugar de intentar gestionar el surgimiento de nuevas ideas, el enfoque defendido con mayor frecuencia por las empresas es fomentar la generación de un gran número de ideas. Quiere decir que se debe desarrollar un modelado conceptual aplicado. Por tanto Galindo, (2015), en su investigación maneja el tema de brechas en gestión tanto tecnológica como innovación en el sector alimentos y bebidas, caracterizándolo como base de investigación de Diseño Mecánico y Desarrollo Industrial en el Macroproyecto, donde el mercado es el que impulsa la tecnología, con la innovación, para así romper las brechas más relevantes, en donde las empresas aplican de manera eficaz y eficiente la gestión de innovación y tecnología. Con respecto a los tiempos en los procesos, las hipótesis específicas 2, 3 y 4; han respondido que existen diferencias significativas entre el grupo de control versus experimental, siendo así, otros estudios referentes a la investigación como es el caso de Valderrama, (2018) en su investigación sostiene que aplicar la filosofía Lean Manufacturing es favorable en las empresas al estar encaminada directamente a mejorar la competitividad creando el máximo valor agregado al consumidor. Para Távara (2017); siguiendo la Metodología PMBOK, llega a la concluir que las acciones por medio de un cronograma de tiempo, se efectúa un manejo y supervisión de lo que lleva a aminorar el periodo a 6 días al planificado. Recíprocamente, San Román y Valdizán (2016) en su investigación trabajó el tema de evaluación del modelo financiero y estratégico, encontrando como resultado que la paralización de la economía restringió el poder adquisitivo de la población aminorando la contingencia de compra, llegando a ultimar que la publicidad efectuada por el gobierno sobre esta temática y el bajo mandato 
conseguido de la población incurrió de manera negativa en la velocidad de ventas entre los meses de marzo a noviembre del año estudiado. Por otra parte Contreras y Palacios (2017) en su investigación analizaron la rentabilidad de las empresas del sector industrial, encontrando que la rentabilidad financiera, declinaron de manera considerada por la baja rotación de sus inventarios, lo cual fortifica la existencia del vínculo adverso entre la liquidez corriente y la rentabilidad financiera.

\section{CONCLUSIÓN O CONSIDERACIONES FINALES}

La Implementación de un sistema de planificación influye de manera significativa en el desarrollo de nuevos productos de una empresa de golosinas, Periodo 2020, según resultados obtenidos por la media del grupo control $(80,56)$ es menor que la media del grupo experimental $(117,30)$, con la prueba T-Student $=-18,219$, y un p_valor $=$ $0.048<0.05$, demostrando un nivel de significancia positivo, siendo aceptada la hipótesis de investigación alterna, de acuerdo a sus indicadores estrategia, exploración, ejecución y entrega.

No se cumple con el objetivo específico 1 dado que ambos exponentes contribuyeron de manera explícita en relación con la influencia del sistema de Estrategia en fijación de tiempos de entrega en una empresa de golosinas, Periodo 2020. Asimismo, de forma individual los métodos que expusieron fueron convincentes para los directivos de la familia de proyectos priorizando el tiempo de atención a los clientes. Sin embargo, se realizó un estudio histórico del tiempo mínimo y máximo de desarrollo de nuevos productos por su tipo y familia, según el indicador fijación de tiempos de entrega.

Existe influencia significativa con el sistema de Exploración en el tiempo de desarrollo y cantidad de procesos de un proyecto en una empresa de golosinas, Periodo 2020. Este resultado responde al sistema de planificación de proyectos y garantiza que se concreten todos los proyectos, disminuyendo la tasa de proyectos demorados de $68.57 \%$ a un $21.4 \%$ y aumentando la cantidad de proyectos terminados de $27.14 \%$ a un $78.57 \%$ (Figura 20). Existe influencia del sistema de Ejecución en la optimización y estandarización de procesos, en una empresa de golosinas, Periodo 2020. Dado que el sistema de planificación no solo se entregan una mayor cantidad de proyectos, de $28.57 \%$ a un $20 \%$ alcanzando un performance del 100\%, optimizando el tiempo de entrega de los proyectos terminados. Por otro lado, el $79 \%$ de los proyectos se entregaron a tiempo y solo el $21 \%$ presento un retraso en la entrega. 
Existe influencia significativa del sistema de Entrega en tiempo de atención y cantidad de proyectos terminados en una empresa de golosinas, Periodo 2020. según resultados obtenidos por la media del grupo control $(12,44)$ es menor que la media del grupo experimental $(20,84)$, con la prueba T-Student $=20,531$, y un p_valor $=0.000<0.05$, demostrando un nivel de significancia positivo, siendo aceptada la hipótesis de investigación alterna, según el indicador tiempo de atención, para el incremento de nuevos proyectos futuros.

Se evidencia la viabilidad la Implementación del sistema de planificación influye de manera significativa en el desarrollo de nuevos productos de una empresa de golosinas de la implementación, durante el periodo 2020, por su impacto, obteniendo una ganancia de $\mathrm{S} / 32,483.57$, con una tasa interna de retorno de $41.97 \%$ y un beneficio costo de 2.3 , es decir por cada sol invertido, se obtienen 2.3 soles de ganancia al finalizar la manufacturación para el siguiente periodo.

\section{LISTA DE REFERENCIAS}

Cabrera, L. (2018). Plan de empresa para una prductora de jugo de fruta natural en la ciudad de Bogotá. (Tesis de grado, Universidad de la Salle). https://ciencia.lasalle.edu.co/cgi/viewcontent.cgi?article=1208\&context=administra cion_agronegocios

Contreras, R., \& Palacios, F. D. (2017). Rentabilidad financiera y liquidez corriente de las empresas del sector industrial que negocian en la Bolsa de Valores de Lima, 20112014. Revista de Investigación Universitaria, 4(2). https://revistas.upeu.edu.pe/index.php/riu/article/view/745

Cruz, D., Quea, J., Bacilio, L., Lizárraga, M., \& Guerra, O. (2018). Buenas prácticas en gestión de manufactureras utilizando la metodología lean manugacturing en las empresas de consumo masivo en el Perú. (Tesis de maestría, Pontificia Universidfad Católica del Perú). http://tesis.pucp.edu.pe/repositorio/bitstream/handle/20.500.12404/12660/QUEA_B ACILIO_PRACTICAS_MANUFACTURA.pdf?sequence=1\&isAllowed=y

Estrada, R. (2015). Análisis de la gestión de proyectos a nivel mundial. Palermo Business Review(12), 61-98. https://www.palermo.edu/economicas/cbrs/pdf/pbr12/BusinessReview12_02.pdf 
Gómez, R., Yepes, C., Rodríguez, F., Roldán, P., Velásquez, W., Lopera, J., . . Agudelo, S. (2009). Manual de Gestión de Proyectos (1 ed.). Universidad de Antioquia. https://todoproyecto.files.wordpress.com/2020/08/manual_gestion_proyectos.pdf

Hernández, R., Fernández, C., \& Baptista, M. (2010). Metodología de la investigación.

$\mathrm{Mc}$

Graw

Hill.

https://www.academia.edu/25455344/Metodolog\%C3\%ADa_de_la_investigaci\%C 3\%B3n_Hernandez_Fernandez_y_Baptista_2010_

Hito Master DAP. (2021). Gestión del tiempo del Proyecto. https://uvmdap.com/programa-desarrollado/bloque-i-el-ciclo-de-vida-del-proyecto/modulo3-planificacion-del-proyecto/gestion-del-tiempo-del-

proyecto/\#el_proceso_de_la_gestion_del_tiempo_del_proyecto

Lavaggi, L., Mori, G., \& Rozas, M. A. (2016). Plan de negocio para una empresa de exportación de chocolate orgánico. (Tesis de maestría, Universidad del Pacífico). https://repositorio.up.edu.pe/bitstream/handle/11354/1520/Luis_Tesis_maestria_20 16.pdf? sequence $=1$

Lopera, M. (2014). Aspectos históricos y epistemológicos de la planificación para el desarrollo. Revista Gerencia y Políticas de Salud, 13(26), 28-43. http://www.scielo.org.co/pdf/rgps/v13n26/v13n26a03.pdf

Martel, F., Peña, E., Ramos, I., \& Silva Santisteban, O. (2015). Plan estratégico de Alicorp SAA del 2015 a 2025. (Tesis de maestría, Pontificia Universidad Católica del Perú).

http://tesis.pucp.edu.pe/repositorio/bitstream/handle/20.500.12404/14961/PE\%C3\% 91A_RAMOS_PLAN_ALICORP\%20S.A.A.pdf?sequence=1\&isAllowed=y

Organización Internacional del Trabajo. (2016). Mejore su negocio: planificación $\begin{array}{llll}\text { empresarial (1a } & \text { ed.). Publicaciones }\end{array}$ https://www.ilo.org/wcmsp5/groups/public/---ed_emp/---emp_ent/--ifp_seed/documents/instructionalmaterial/wcms_553924.pdf

Pérez, E. (2016). Propuesta para mejraor el tiempo de entrega en una industria manufacturera metalmecánica. (Monografía de grado, Universidad de San Buena Ventura Medellín). http://bibliotecadigital.usbcali.edu.co/bitstream/10819/3637/1/Propuesta_Entrega_I ndustria_Perez_2016.pdf 
Salinas, K. (Junio de 2016). Estudio de factibilidad para la creación de una empresa dedicada a la industrialización y comercialización de chocolate raw orgánico y ecológico en el cantos vinces. (Tesis de grado, Universidad de Guayaquil). http://repositorio.ug.edu.ec/bitstream/redug/11296/1/TESIS\%20KARLA\%20SALI NAS\%20CORONEL.\%20CHOCOLATE\%20RAW\%20junio2016.pdf

San Román Guerra, R., \& Valdizán Martínez, J. (2016). Evaluación del modelo financiero $y$ del modelo de estrategia comercial de un proyecto inmobiliario mixto en Magdalena del Mar. (Tesis de maestría, Universidad Católica del Perú). http://hdl.handle.net/20.500.12404/7574

Secretaría de la Función Pública. (2016). Guía para la optimización, estandarización y mejora continua de procesos. https://www.gob.mx/cms/uploads/attachment/file/56904/Gu_a_para_la_Optimizaci _n_Estandarizaci_n_y_Mejora_Continua_de_Procesos.pdf

Távara, J. (2017). Gestión del Proyecto de Reubicación del Data Center y Centro de Control en el Área de Seguridad Electrónica en Minera Yanacocha Siguiendo la Metodología PMBOK en lo Referente en Tiempo y Costo. (Tesis de maestría, Universidad Privada Antonio Guillermo Urrelo). http://repositorio.upagu.edu.pe/bitstream/handle/UPAGU/387/17.\%20TESIS\%20\%20T\%c3\%81VARA\%20CARBAJAL.pdf?sequence=1\&isAllowed=y

Umaña, F. (2016). Guía para la gestión del alcance, tiempo y costo de los proyectos de Desarrollos Mega. (Proyecto de grado, Instituto Tecnológico de Costa Rica). https://repositoriotec.tec.ac.cr/bitstream/handle/2238/10042/guia_para_gestion_alca nce_tiempo_costo_proyectos_desarrollos_mega.pdf?sequence=1\&isAllowed=y

Valderrama, M. (01 de Junio de 2018). Propuesta de mejora para la reducción de tiempos en el proceso productivo para uvas de mesa variedad Red Globe aplicando herramientas Lean Manufacturing. (Tesis de grado, Universidad Peruana de Ciencias Aplicadas). https://repositorioacademico.upc.edu.pe/bitstream/handle/10757/624262/VALDER RAMA_LM.pdf?sequence $=1 \&$ isAllowed $=\mathrm{y}$

Varela, A. (2015). Adopción de métodos, técnicas y herramientas para la innovación: framework en función de casos reales. (Tesis de doctorado, Universidad Politécnica de Catalunya). https://core.ac.uk/download/pdf/78444632.pdf 
Vera, S. (16 de Julio de 2018). Propuesta de un sistema de planificación y contro de la producción para la empresa Fabrication Technology Company SAC para mejorar el nivel de servicio. (Tesis de grado, Universidad Católica Santo Toribio de Mogrovejo).

http://tesis.usat.edu.pe/bitstream/20.500.12423/1371/1/TL_VeraCubasSharon.pdf

Vilcapoma, J. (2016). Aplicación del sistema de planificación last planner y su influencia en la gestión operativa del proyecto de mejoramiento y modificación de la línea de carga concentrado en el patio ferroviario de la sociedad minera el Brocal. (Tesis de grado, Universidad Continental). https://repositorio.continental.edu.pe/bitstream/20.500.12394/3368/1/INV_FIN_10 5_TE_Vilcapoma_Romero_2016.pdf 